\title{
Reasons for variations in the use of open access gastroscopy by general practitioners
}

\author{
A P S Hungin, M G Bramble, H O’Callaghan
}

\section{Abstract}

This study aimed to investigate the wide variation between general practitioners (GPs) in their use of open access gastroscopy by assessing (i) their partnership share, workload, and the aggregated practice request rate; (ii) correlations with their professional and practice characteristics; and (iii) a comparison with referral rates to medicine, surgery, and all specialities. All 145 GPs and their practice managers were sent a questionnaire and hospital held data on all requests for open access gastroscopy over one year were reviewed. During the year, the 145 GPs made 1210 requests for open access gastroscopy, varying from one to 44 per GP. There were 987880 practice consultations altogether, an average of 22451 per practice or 7127 per whole time practitioner. Requests for open access gastroscopy formed $2 \cdot 4 \%$ of all referrals, an average of one per 1000 consultations, or eight per GP. Of a total of 49123 referrals to all specialities (371 per GP) $4218(8 \cdot 5 \%)$ were to medicine, and 6444 to surgery $(13 \cdot 1 \%)$. The following factors did not correlate with requests: vocational training, a concurrent hospital post, length of service, or receipt of the deprivation allowance by the practice. When the open access gastroscopy referral rate was aggregated for each practice the variation between practices was narrowed to essentially twofold. Requests for open access gastroscopy form a small proportion of all referrals $(2 \cdot 4 \%)$. Aggregated practice request rates are relatively uniform compared with the wide variation between individual GPs, suggesting a disproportionate gastroenterology workload between partners. The open access gastroscopy service does not seem to be subject to misuse from most GPs if a variation in practice usage is used as a measure.

(Gut 1995; 36: 180-182)

Keywords: open access gastroscopy.

Data on open access gastroscopy (OAG) usage suggest wide differences in the uptake of the service, with as much as a 40 -fold variation between general practitioners (GPs). ${ }^{1}$ Fears that this variation may mask inappropriate use had led to a reluctance by some specialists to offer OAG. ${ }^{2}$ These data have been based on referral numbers alone, however, with no attempt to standardise for practice workload or whether the GPs were full or part time. In addition, it is difficult to assess the importance of any variations in OAG requests without information on referrals to other specialities for comparison.

This study aimed to interpret individual GP's requests for OAG in relation to their partnership equivalent, workload, and their practices' aggregated request rate. We also examined the GP's professional and practice characteristics to see if they correlated with OAG request numbers, and made a comparison with their referral rates to medical and surgical specialities.

\section{Methods}

All the GPs in the South Tees Health District, where OAG had been operating for three years, were sent two postal questionnaires. The first requested information on professional characteristics: age, sex, years in practice, whether vocationally trained, if a hospital appointment was also held, and their partnership equivalent, for example, if they worked half-time. The second questionnaire was directed to the practice manager and sought information about the list size, number of partners, the total number of consultations with all the partners during the preceding year and details of practice referral numbers to medicine, surgery, and to all specialities combined. Most of these details are required for the preparation of the statutory annual practice report and are easily accessible.

Information on the individual GP's request numbers to OAG for the entire health district was obtained from the computerised records held at Middlesbrough General Hospital.

The questionnaires were structured and analysed using Epi-Info software and its associated statistical package.

\section{Results}

\section{PRACTICES AND PRACTITIONERS}

The South Tees Health District has a population of 289000 and is served by 145 GPs. Of these, 128 cooperated in the survey (response rate $88 \%$ ), comprising 44 practices with an average partnership equivalent of $3 \cdot 15$. Three practices did not respond.

There was a statistically significant correlation between the number of OAG requests from the practice and the list size (correlation coefficient $\mathrm{r}^{\wedge}=0.58, \mathrm{p}=<0.0001$ ) and partnership size $\left(r^{\wedge}=0.59, p=<0.0001\right)$, but not
Dr A P S Hungin, The Health Centre, Eaglescliffe, Stockton on Tees

Accepted for publication 27 May 1994 
Referrals to hospital from 987880 GP consultations during one year (1991-92)

\begin{tabular}{lccc}
\hline & \multicolumn{3}{c}{ Average } \\
& No & per GP & Percentage \\
\hline All specialties & 49123 & 371 & 100 \\
Medicine & 4218 & 30 & $8 \cdot 5$ \\
Surgery & 6444 & 47 & $13 \cdot 1$ \\
Open access gastroscopy & 1210 & $8 \cdot 3$ & $2 \cdot 4$ \\
\hline
\end{tabular}

whether the practice received the deprivation allowance (58\% of practices).

Altogether $70 \%$ of the GPs were vocationally trained and $24 \%$ had a concurrent hospital appointment (none in gastroenterology). The average length of service in practice was 13 years (range 1-35 years). None of these factors correlated with the variation in referral numbers.

\section{CONSULTATIONS}

The total number of consultations with all the GPs during the year was 987880 , giving an average of 22451 per practice or 7127 per partner equivalent. These figures do not include consultations for antenatal clinics, immunisations, or consultations with the non-GP members of the primary health care

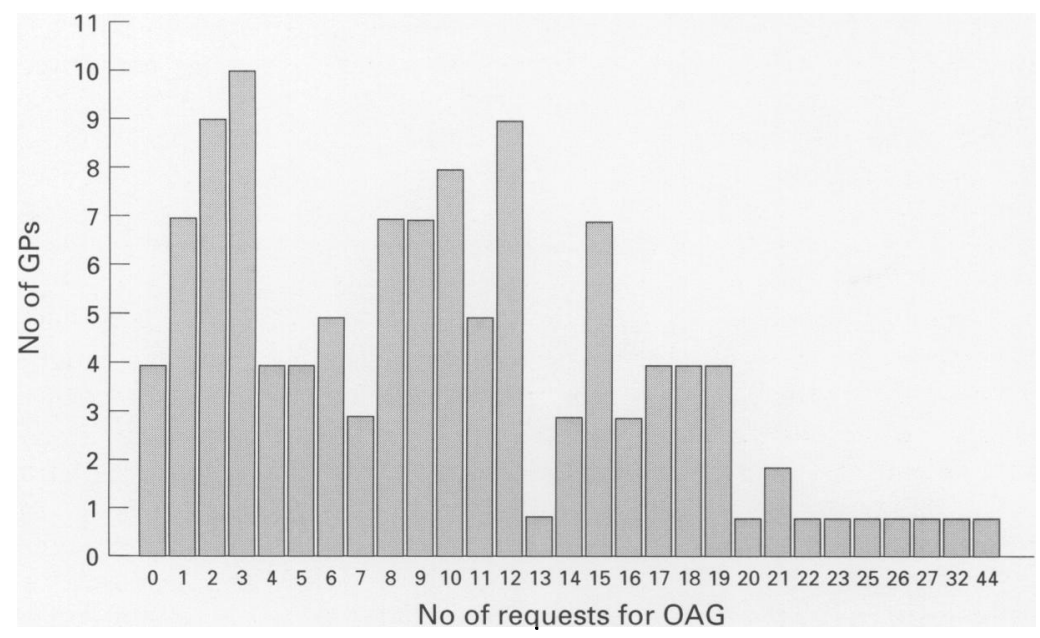

Figure 1: Number of requests for open access gastroscopy (OAG) made by general practitioners (GPs) in 1991-92.

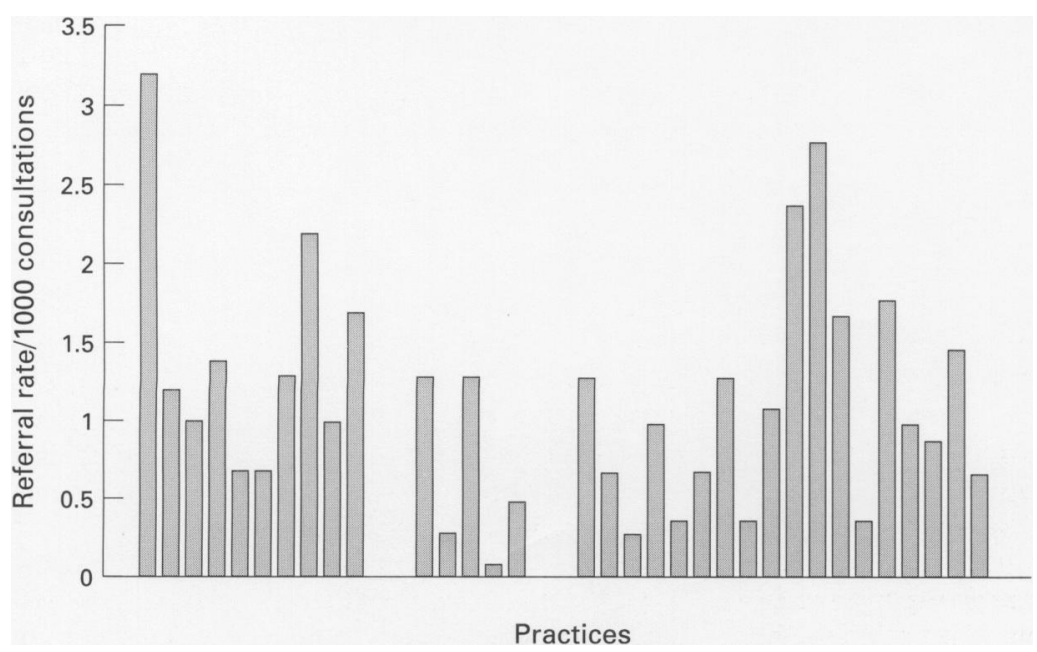

Figure 2: Referral rate for open access gastroscopy (per 1000 consultations) determined from practice data from 37 practices (mean one referral/1000 consultations). team. Based on the estimate of $2-3 \%,{ }^{3}$ approximately 25000 consultations were for upper gastrointestinal symptoms.

\section{REFERRALS}

The total number of referrals to all specialities was 49123 , an average of 371 per partner equivalent (Table). Referrals to 'medicine' accounted for $8.5 \%$ of the total (30 per partner equivalent) and those to 'surgery' $13 \cdot 1 \%$ (47 per partner equivalent). In comparison, there was a total of 1210 requests for OAG $(2 \cdot 4 \%$ or 8.3 per partner equivalent).

The number of OAG requests per GP (Fig 1) varied from nil (12 GPs) to 44 (one GP).

When the referrals were aggregated per practice and expressed as a ratio of 1000 consultations per year, the variation was reduced essentially to a narrow band between $0 \cdot 7-1 \cdot 3$ referrals per 1000 consultations per year (Fig 2), with a few exceptions. One practice registered a figure of 3.0 and four registered only 0.4 requests per 1000 consultations per year. Four practices did not use the service. The average referral rate was 1 per 1000 consultations.

\section{Discussion}

A potential source of error is in the way in which practices record their consultation and referral numbers. Although these are required for the annual practice report there is not yet a standard recording format. Nonetheless, during this study collection it became apparent that there was relative consistency of figures between the practices, and we believe that these figures are as accurate as currently available. As recording systems become more uniform it may be possible to reduce errors due to such variations.

At first sight there seems to be a wide variation of up to 40 fold between GPs in the number of requests for OAG. However, aggregated practice request rates, corrected for workload, are remarkably uniform as opposed to the individual referral numbers. What variation is demonstrated (essentially twofold) is less than the fourfold or more variation expected from national data on all referrals. ${ }^{4}$

The results also show that requests for OAG form a small proportion of all referrals $(2 \cdot 4 \%)$ and represent a tiny proportion of the total gastrointestinal throughput for the GP. From an estimated 25000 consultations for upper gastrointestinal problems there were 1210 OAG requests $(4 \cdot 8 \%)$.

One possible explanation is that partners within a practice have differing exposures to upper gastrointestinal problems, with some carrying a disproportionate workload in gastroenterology. This is likely to be related to a degree of intra-practice referral, self selection by patients, and the personal and clinical characteristics of the doctor. Two of the four highest users of the service were single handed practitioners but the specifically studied 
practice and professional characteristics of the GPs were not determinants of the rate of usage.

The findings do not bear out the fear that OAG services may be subject to abuse by most GPs. Data on this service usage should be interpreted on the basis of practices and workload rather than individual request numbers. To gain a better understanding of the demand for OAG, more research is needed to determine the reasons and decision making factors during the consultation process.
We would like to thank the participating practices, the Cleveland Primary Care Research Panel for financial assistance and the Northern Research Network (NoReN).

1 Bramble MG, Cooke WM, Corbett WA, Cann PA, Clarke $\mathrm{D}$, Contractor B, et al. Organising unrestricted open access gastroscopy in South Tees. Gut 1993; 34: 422-7.

2 Holdstock $G$, Wiseman $M$, Loehry CA. Open access endoscopy service for general practitioners. $B M \ngtr 1979 ; 41$ : 438-44.

3 Government Statistical Services. Morbidity statistics from general practice, third national study 1981-82. London: HMSO, 1986: $99,157$.

4 Roland R. Referrals to medical outpatients. London: Royal College of Physicians Publications, 1992: 34. 